\title{
Anticoagulants and cancer mortality in the Finnish randomized study of screening for prostate cancer
}

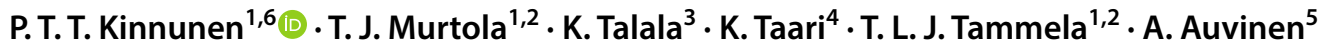

Received: 11 December 2018 / Accepted: 12 June 2019 / Published online: 17 June 2019

(c) The Author(s) 2019

\begin{abstract}
Purpose Anticoagulants may reduce mortality of cancer patients, though the evidence remains controversial. We studied the association between different anticoagulants and cancer death.

Methods All anticoagulant use during 1995-2015 was analyzed among 75,336 men in the Finnish Randomized Study of Screening for Prostate Cancer. Men with prevalent cancer were excluded. Multivariable Cox regression was performed to compare risk of death from any cancer and disease-specific death from 9 specific cancer types between (1) anticoagulant users overall and (2) warfarin users compared to anticoagulant non-users and (3) warfarin or (4) low-molecular-weight heparins (LMWH) compared to users of other anticoagulants. Medication use was analyzed as time-dependent variable to minimize immortal time bias. 1-, 2- and 3-year lag-time analyses were performed.

Results During a median follow-up of 17.2 years, a total of 27,233 men died of whom 8033 with cancer as the primary cause of death. In total, 32,628 men (43\%) used anticoagulants. Any anticoagulant use was associated with an increased risk of cancer death ( $\mathrm{HR}=2.50,95 \% \mathrm{CI} 2.37-2.64)$ compared to non-users. Risk was similar independent of the amount, duration, or intensity of use. The risk increase was observed both among warfarin and LMWH users, although not as strong in warfarin users. Additionally, cancer-specific risks of death were similar to overall cancer mortality in all anticoagulant categories. Conclusion Our study does not support reduced cancer mortality among anticoagulant users. Future studies on drug use and cancer mortality should be adjusted for anticoagulants as they are associated with significantly higher risk of cancer death.
\end{abstract}

Keywords Cancer mortality $\cdot$ Anticoagulant $\cdot$ Warfarin $\cdot$ Low-molecular weight heparins $\cdot$ Cohort

Electronic supplementary material The online version of this article (https://doi.org/10.1007/s10552-019-01195-x) contains supplementary material, which is available to authorized users.

P. T. T. Kinnunen

Kinnunen.Pete.T@student.uta.fi

T. J. Murtola

Teemu.Murtola@uta.fi

K. Talala

Kirsi.Talala@cancer.fi

K. Taari

kimmo.taari@helsinki.fi

T. L. J. Tammela

Teuvo.Tammela@uta.fi

A. Auvinen

Anssi.Auvinen@staff.uta.fi

\section{Introduction}

Venous thromboembolism (VTE) is a clinically important complication among patients with malignancies, as the risk of VTE is five- to sevenfold in patients with cancer [1,2]. However, especially tumors of pancreas, brain, liver, and

1 School of Medicine, University of Tampere, Tampere, Finland

2 Department of Urology, Tampere University Hospital, Tampere, Finland

3 Finnish Cancer Registry, Helsinki, Finland

4 Department of Urology, University of Helsinki and Helsinki University Hospital, Helsinki, Finland

5 Department of Epidemiology, University of Tampere, Tampere, Finland

6 Faculty of Medicine and Life Sciences, University of Tampere, Lääkärinkatu 1, 33520 Tampere, Finland 
lung are associated with even more risk of VTE and additionally the risk increases considerably with more advanced disease [3]. Cancer patients with deep VTE or pulmonary embolism have an eightfold risk of death compared to VTE in patients without cancer [4]. Prognosis for cancers patients with embolism is fairly poor: 1-year overall survival is only $38 \%$ [5].

The biological rationale of anticoagulants is against cancer progression rather than cancer initiation in experimental animal studies. Evidence against cancer development is limited. It has been reported that tissue factor pathway inhibition has been reported to be relevant in formation of certain brain tumors [6]. It has further been suggested that cancer cells may activate coagulation through increased expression of tissue factor increasing malignant phenotype of cancer cells [7].

Significantly more evidence is found on cancer progression and coagulation cascade as well as anticoagulants; infusion of small amounts of thrombin increases colon cancer metastases [8]. Hemophilic mice with Factor VIII deficiency are protected against experimentally induced cancer cell metastasis [9] and in heterozygous prothrombin-deficient mice, metastatic spread is considerably reduced [10]. In contrast, in hypercoagulable mice, risk of metastases is increased [11]. Concordantly, anticoagulants targeting Factor $\mathrm{Xa}$ and/or thrombin reduce metastases and increases survival in animal studies $[12,13]$. Figure 1 illustrates aforementioned associations reported in the literature.

Vitamin $\mathrm{K}$ antagonists (VKA) such as warfarin have been promising in some experimental studies [14, 15], but a systematic review covering five randomized controlled trials (RCTs) (from 1984 to 1997) indicated no evidence for improved survival among cancer patients [16]. Results of RCTs on low-molecular weight heparins (LMWH) and cancer survival are inconsistent and considerable number of included patients had at least Stage III disease possibly affecting the generalizability to less advanced disease [17-20]. Due to improvements in contemporary cancer care since the 1990s, it is unclear whether the results from the warfarin RCTs are still applicable. Thus, the effect of anticoagulants on risk of cancer death is still under debate.

To date only three cohort/case-control studies of $>300$ patients have assessed warfarin use and risk of cancer-specific death [21-23] and only one covers other anticoagulant drugs [23]. Due to paucity of studies on this topic, we explored the association between all types of anticoagulants during 1996-2015 and cancer mortality in the population of the Finnish Randomized Study of Screening for Prostate Cancer (FinRSPC) [24].

\section{Methods}

\section{Study cohort}

FinRSPC includes 80,458 men aged 55-67 years at baseline. After exclusion of prevalent prostate cancer cases, the men were randomized during 1996-1999 either to prostatespecific antigen (PSA) screening at 4-year intervals or to no intervention. For this analysis, we further excluded 3,279 men with previous diagnoses of any other cancer types at baseline. In total, 75,336 men were included in the analysis. This is demonstrated in Supplementary Fig. 1. Information on baseline cancers was obtained from comprehensive Finnish cancer registry, which covers over $90 \%$ of cancer cases diagnosed in Finland. The follow-up started at randomization and continued until death, emigration from Finland or 1 January 2016, whichever occurred first.

Information on deaths was obtained from the national death certificate registry of Statistics Finland, which assigns official causes of death based on mandatory death certificates. The information included date and immediate,
Fig. 1 Simplified illustration of relation between coagulation cascade and cancer metastasis and mortality in the literature. Additionally, antimetastatic effect of anticoagulant drugs reported in the literature that may decrease cancer mortality

\section{Supporting metastatic spread:}

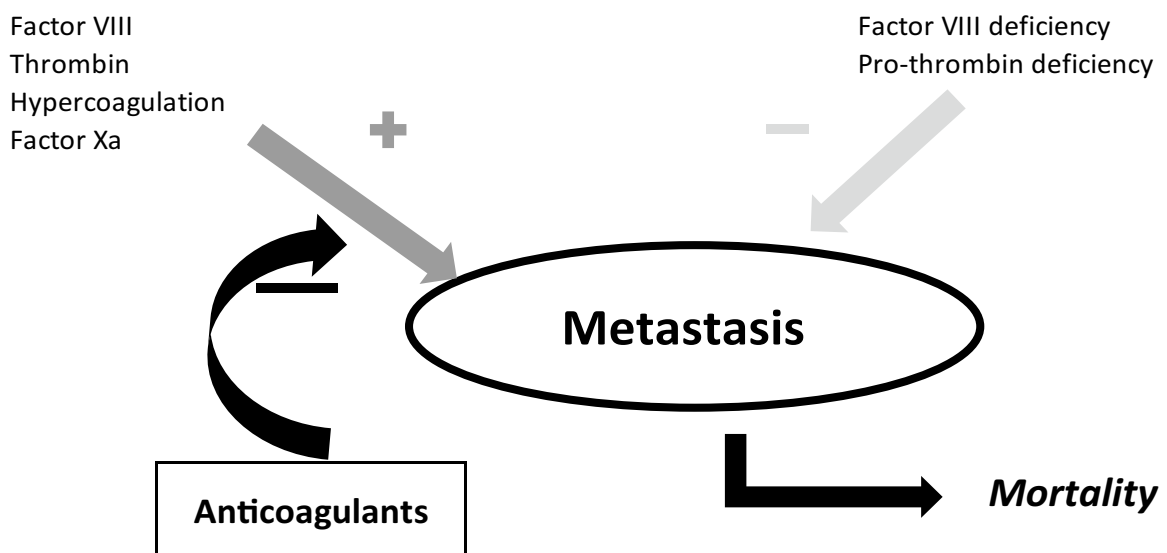


primary, and contributory causes of death. Only deaths with cancer (ICD-10 codes C00-D48) listed as the primary cause of death were regarded as cancer deaths.

We also obtained information on conditions that are major indications for anticoagulant use: pulmonary embolism (ICD-10: I26.0, I26.9), venous thromboembolism (ICD-10: I82.0-82.9), and atrial fibrillation (ICD-10: I48) from the national care register for health care (HILMO) maintained by the National Institute for Health and Welfare, which covers all hospitals in Finland and records all diagnoses for in- and out-patients visits during 1996-2014. Diagnoses from primary care are not covered. Additionally, Charlson Comorbidity Score [25] was calculated based on diagnoses recorded in the HILMO database during 1996-2015.

\section{Information on anticoagulant usage}

Information on anticoagulant drug purchases during 1996-2015 was obtained by linking the study cohort to a national medication reimbursement database maintained by the Finnish Social Insurance Institution (SII). The record linkage was based on the unique personal identification number assigned to all residents of Finland. Medication usage data were available for 75,336 men. As a part of the national health insurance that covers all Finnish citizens, SII provides reimbursements for purchases of physician-prescribed drugs. In Finland, anticoagulant drugs are available only through physicians' prescription. Therefore, the obtained data cover all anticoagulant reimbursements in outpatient setting. Drugs used during hospital inpatient periods are not covered.

All 14 anticoagulant drugs used in outpatient setting during the study period were identified based on their Anatomic Therapeutic Chemical (ATC) codes. These were warfarin (B01AA03) and fenindion (B01AA02) as VKAs, dalteparin (B01AB04), enoxaparin (B01AB06), and tinzaparin (B01AB10) as heparins, clopidogrel (B01AC04), dipyridamole (B01AC30), iloprost (B01AC11), and ticlopidine (B01AC05) as aggregation inhibitors, dabigatran (B01AE07) and ximelagatran (B01AE05) as direct thrombin inhibitors, as well as factor Xa inhibitors rivaroxaban (B01AX06), apixaban (B01AF02), and fondaparinux (B01AX05). Additionally, we obtained information on cholesterol-lowering drugs, aspirin and other non-steroidal antiinflammatory drugs (NSAIDs), antihypertensive drugs, and antidiabetic drugs as these may influence survival [26-30].

\section{Statistical analysis}

Cox proportional hazards regression was used to calculate hazard ratios (HR) and 95\% confidence intervals (CIs) for cancer death. The follow-up started at the FinRSPC randomization and continued until death or right censoring (emigration from Finland or 1 January 2016), whichever occurred first. Time metric was years and months since the baseline. Validity of proportional hazards assumption was tested with an interaction term between follow-up time and time-fixed variables. In each case, the interaction term was statistically non-significant, confirming the assumption. In addition to general cancer mortality, we explored cancer-specific mortality separately for the following cancer types: lung, gastric, colorectal, central nervous system (CNS), non-Hodgkin lymphoma, hepatic, pancreatic, renal, and bladder. Prostate cancer deaths are included in general cancer mortality; prostate cancer-specific mortality has been covered in detail in our previous study [23].

For each man in the study cohort, yearly medication purchases were summed to obtain the total annual amount. Differences in dosing between different anticoagulants were standardized by dividing the total annual milligram amount with the standard Defined Daily Dose (DDD) as listed by the WHO [31]. Each year with registered anticoagulant purchases was regarded as a year of usage regardless of the amount. Average yearly dosage (intensity of use) was estimated by dividing the number of annual doses with the number of years of usage. Intensity of use was also updated annually as a time-dependent variable.

Anticoagulant use after the FinRSPC randomization was analyzed as a time-dependent variable: medication use status and cumulative usage were updated for each year of follow-up based on annual drug purchases. Men were categorized as non-users until the year of the first anticoagulant purchase. The status was changed into a user after the first purchase and maintained as a user for each year with recorded purchases. Men who discontinued anticoagulant purchases during the follow-up were categorized as previous users. In ever-users, both current and previous users of anticoagulants were included. Similarly, cumulative amount, duration, and average yearly dose were updated for each follow-up year according to yearly anticoagulant purchases.

In the analysis comparing warfarin users with men using other anticoagulant drugs, the men were categorized as warfarin users each year with recorded warfarin purchases even if they had used other types of anticoagulants. Only for years with recorded anticoagulant purchases without warfarin use, men were considered warfarin non-users. Similar methodology was used for comparison between heparin users and men using non-heparin anticoagulants.

The main analysis was performed by adjusting Cox regression model for age (continuous variable), use of other medications (categorical variables), indications for anticoagulant usage (categorical variables), and Charlson Comorbidity Score (continuous variable) to calculate hazard ratios (HR) and 95\% confidence intervals (CIs) for cancer deaths. The analysis was performed separately for (1) all anticoagulants as a group, (2) warfarin users 
compared to anticoagulant non-users, (3) warfarin users, and (4) LMWH users compared to users of other anticoagulant drugs.

In order to evaluate impact of timing of anticoagulant use, we performed a lag-time analysis, where anticoagulant exposure was lagged 1-3 years forward in follow-up time, i.e., outcome events were related to $1-3$ years earlier.

Additionally, we performed subgroup analyses stratified by age at randomization, indication for anticoagulant drug use, Charlson Comorbidity Score divided into 3 groups (0 points, 1-2 points, and 3 or more points), and use of other drugs as listed earlier. We also stratified the subgroup analyses by Body Mass Index (BMI), which was available for 11,345 men of the study population. Statistical significance of the effect modification by background variables was tested by adding an interaction term between anticoagulant use and the tested variable in the Cox regression analysis to see whether it improved model fit. $p$ value $<0.05$ was considered statistically significant.

All statistical analyses were carried out using IBM SPSS Statistics 22.0. All statistical tests are two-sided.

\section{Results}

\section{Population characteristics}

During a median follow-up of 17.2 years, 27,233 men died, of whom 8,033 with cancer as the primary cause of death. Among men who died from cancer, the median follow-up was 11.3 years. A total of 48,103 men were followed up until 1 January 2016. In total, 32,628 men (43\%) had used anticoagulants during 1995-2015. Distribution of cancer mortality, numbers of cancer-specific deaths, indications for anticoagulant usage, use of other medication, and other background variables stratified by anticoagulant user status are presented in Table 1.

\section{Risk of cancer death by use of any anticoagulants}

Ever-use of any anticoagulant was associated with an increased risk of cancer death $(\mathrm{HR}=2.50,95 \%$ CI 2.37-2.64). The risk was increased for both current $(\mathrm{HR}=2.20,95 \% \mathrm{CI} 2.06-2.35)$ and previous users $(\mathrm{HR}=2.81,95 \% \mathrm{CI} 2.64-2.99)$. The risk increase was similar regardless of the amount or intensity of use. The increased risk was most pronounced in high-intensity (more than $207 \mathrm{DDD} /$ year) usage (HR $=3.09,95 \%$ CI 2.87-3.34). When exploring cancer-specific risk of death, the risk was elevated for all cancer types, being lowest for hepatic cancer and highest for bladder cancer (Table 3).

\section{Risk of cancer death in relation to warfarin use}

Compared to other anticoagulants, use of warfarin was associated with a significantly lower risk of cancer death (HR $=0.45,95 \%$ CI 0.41-0.50). This was observed regardless of the amount, duration, or intensity of use (Table 2), the risk decrease being most apparent in high-dose (cumulative use greater than $1,200 \mathrm{DDD}, \mathrm{HR}=0.36,95 \% \mathrm{CI} 0.31-0.43$ ) and high-intensity (more than $193 \mathrm{DDD} /$ year, HR $=0.31$, 95\% CI 0.27-0.37) use of warfarin. The risk of cancerspecific death was significantly lower among warfarin users compared to users of other anticoagulants in all cancer types (Table 3).

When using anticoagulant non-users as the reference instead of men using other types of anticoagulants, risk for cancer death was significantly increased also among warfarin users $(\mathrm{HR}=2.34,95 \% \mathrm{CI} 2.20-2.48)$. The risk increase was considerably elevated for previous users $(\mathrm{HR}=3.01$, 95\% CI 2.83-3.21) and moderately increased among current warfarin users compared to anticoagulant non-users ( $\mathrm{HR}=1.31,95 \% \mathrm{CI} 1.19-1.43)$. Warfarin use was associated with an elevated risk of cancer death compared to anticoagulant non-users regardless of amounts, duration, or intensity of use (Table 2). Risk of cancer-specific death was elevated for all cancer types (Table 3).

\section{Risk of cancer death related to heparin use}

In total, 12,326 men used LMWH during the study period (69\% had used 80 DDD or less). LMWH users compared to non-LMWH anticoagulant users were at significantly higher risk of cancer death (HR 2.04, 95\% CI 1.90-2.20) (Table 2). Risk increase was especially high for high dose (more than $80 \mathrm{DDD}$ ), use for 2 year or longer, and highintensity (more than $60 \mathrm{DDD} /$ year) usage. Low dose (40 DDD or less) was not associated with increased risk of cancer death. Conversely, in 3-year lag-time the risk association was reversed to be lower in LMWH users compared to users of other anticoagulant drugs ( $\mathrm{HR}=0.86,95 \%$ CI $0.77-0.95)$. Cancer mortality was significantly elevated for all examined cancer types with the exception of central nervous system cancers, where the risk increase was statistically non-significant (Table 3). The most pronounced risk increase was observed for pancreatic and colorectal cancer death.

\section{Lag-time analysis}

Any use of anticoagulants compared to non-users was associated with an increased risk of cancer death in 1-year, 2-year, and 3-year lag-time analyses. The risk slightly attenuated with longer lag-time but remained elevated. Risk of cancer-specific death remained elevated in 3-year lag-time for all cancer types (Table 3 ). 
Table 1 Characteristics of the study population stratified by anticoagulant user status

\begin{tabular}{|c|c|c|c|c|c|c|}
\hline & \multicolumn{6}{|c|}{ Anticoagulant user status } \\
\hline & No anticoagulation & Warfarin $^{\mathrm{a}}$ & $\mathrm{LMWH}^{\mathrm{a}}$ & $\begin{array}{l}\text { Non-warfarin or } \\
\text { LMWH anticoagu- } \\
\text { lants }\end{array}$ & Total $n$ of deaths & $\begin{array}{l}\text { Median } \\
\text { age at } \\
\text { death }\end{array}$ \\
\hline$n$ of men in the study population & 42.708 & 17.826 & 12.326 & 8.595 & & \\
\hline $\begin{array}{l}\text { Overall cancer mortality/10,000 } \\
\text { person years } \\
n \text { of cancer deaths }\end{array}$ & 75 & 54 & 81 & 46 & & \\
\hline Overall & $4,766(11.2 \%)$ & $1,549(8.7 \%)$ & $1,733(14.1 \%)$ & $654(7.6 \%)$ & 8.033 & 72 \\
\hline Lung cancer & $1,404(3.3 \%)$ & $380(2.1 \%)$ & $351(2.8 \%)$ & $213(2.5 \%)$ & 1.739 & 71 \\
\hline Gastric cancer & $203(0.7 \%)$ & $52(0.3 \%)$ & $76(0.6 \%)$ & $24(0.3 \%)$ & 327 & 71 \\
\hline Colorectal cancer & $414(1.0 \%)$ & $144(0.8 \%)$ & $233(1.9 \%)$ & $49(0.6 \%)$ & 764 & 72 \\
\hline Central nervous system cancers & $112(0.3 \%)$ & $32(0.2 \%)$ & $27(0.2 \%)$ & $21(0.2 \%)$ & 182 & 69 \\
\hline Non-Hodgkin lymphomas & $125(0.3 \%)$ & $64(0.4 \%)$ & $65(0.5 \%)$ & $18(0.2 \%)$ & 244 & 74 \\
\hline Hepatic cancer & $292(0.7 \%)$ & $79(0.4 \%)$ & $67(0.5 \%)$ & $34(0.4 \%)$ & 441 & 71 \\
\hline Pancreatic cancer & $432(1.0 \%)$ & $118(0.7 \%)$ & $195(1.6 \%)$ & $45(0.5 \%)$ & 733 & 71 \\
\hline Renal cancer & $124(0.3 \%)$ & $51(0.3 \%)$ & $72(0.6 \%)$ & $20(0.2 \%)$ & 239 & 72 \\
\hline Bladder cancer & $87(0.2 \%)$ & $52(0.3 \%)$ & $66(0.5 \%)$ & $18(0.2 \%)$ & 195 & 73 \\
\hline \multicolumn{7}{|l|}{ Recorded diagnoses of } \\
\hline Pulmonary embolisms & $112(0.3 \%)$ & $661(3.7 \%)$ & $634(5.1 \%)$ & $59(0.7 \%)$ & & \\
\hline Venous thromboembolism & $23(0.1 \%)$ & $205(1.2 \%)$ & $233(1.9 \%)$ & $12(0.1 \%)$ & & \\
\hline Atrial fibrillation & $953(2.2 \%)$ & $9,174(51.5 \%)$ & $3,833(31.1 \%)$ & $508(0.6 \%)$ & & \\
\hline \multicolumn{7}{|l|}{ Charlson Comorbidity Score } \\
\hline 0 points & $24,979(58.5 \%)$ & $6,354(35.6 \%)$ & $4,133(33.5 \%)$ & $3,879(45.1 \%)$ & & \\
\hline $1-2$ points & $12,669(29.7 \%)$ & $6,226(34.9 \%)$ & $4,566(37.0 \%)$ & $3,020(35.1 \%)$ & & \\
\hline 3 or more points & $5,060(11.8 \%)$ & $5,246(29.4 \%)$ & $3,627(29.4 \%)$ & $1,696(19.7 \%)$ & & \\
\hline \multicolumn{7}{|l|}{ Use of other medication } \\
\hline Statin users & $16,307(38.2 \%)$ & $11,613(65.1 \%)$ & $7,827(63.5 \%)$ & $7,060(82.1 \%)$ & & \\
\hline Aspirin users & $4,218(9.9 \%)$ & $3,666(20.6 \%)$ & $2,542(20.6 \%)$ & $4,266(49.6 \%)$ & & \\
\hline NSAID users & $34,574(80.1 \%)$ & $16,016(89.8 \%)$ & $11,865(96.3 \%)$ & $7,883(91.7 \%)$ & & \\
\hline Antihypertensive drug users & $27,860(65.2 \%)$ & $17,055(95.7 \%)$ & $10,698(86.8 \%)$ & $7,835(91.2 \%)$ & & \\
\hline Antidiabetic drug users & $6,300(14.8 \%)$ & $4,237(23.8 \%)$ & $3,154(25.6 \%)$ & $2,215(25.8 \%)$ & & \\
\hline \multicolumn{7}{|l|}{ Body Mass Index (BMI) ${ }^{b}$} \\
\hline$<27.6$ & $3,442(8.1 \%)$ & $1,078(6.0 \%)$ & $958(7.8 \%)$ & $604(7.0 \%)$ & & \\
\hline$\geq 27.6$ & $2,780(6.5 \%)$ & $1,603(9.0 \%)$ & $1,265(10.3 \%)$ & $700(8.1 \%)$ & & \\
\hline
\end{tabular}

NSAID non-steroidal anti-inflammatory drug

${ }^{a}$ Warfarin and LMWH user status not mutually exclusive. 6,119 men used both warfarin and LMWH during the study period

${ }^{\mathrm{b}}$ Information on BMI was available only for 11,345 men. Value 27.6 was the median and was used to divide into two groups of equal size

When comparing warfarin users to anticoagulant nonusers, the risk remained elevated although slightly attenuated over all lag times up to 3 years (Table 3 ).

Risk decrease for overall cancer death observed in the main analysis disappeared in the lag-time analyses comparing warfarin users to users of other anticoagulants (Table 2). The same was observed for cancer-specific risks of death, and the risk decrease remained statistically significant for only non-Hodgkin lymphomas in 1- and 2-year lag-time models (Table 3).

\section{Subgroup analysis}

Among all anticoagulant users, significant effect modification was observed for age at randomization, indication for anticoagulant use (excluding atrial fibrillation), for use of statins or antidiabetic drugs, and Charlson Comorbidity Score ( $p<0.001$ for each). Also BMI modified the effect statistically significantly $(p=0.010)$. The effect modification was most pronounced for VTE diagnosis, the risk 
Table 2 Multivariable and lag-time hazard ratios (95\% CI) related to cancer-specific deaths

\begin{tabular}{|c|c|c|c|c|c|}
\hline & $n$ of deaths & Multivariable-adjusted & 1-year lag-time & 2-year lag-time & 3-year lag-time \\
\hline \multicolumn{6}{|c|}{ Any anticoagulant compared to anticoagulant non-users } \\
\hline None & 4.766 & Ref. & Ref. & Ref. & Ref. \\
\hline Ever-users & 3.267 & $2.50(2.37-2.64)$ & $2.20(2.08-2.32)$ & $1.73(1.64-1.83)$ & $1.67(1.57-1.77)$ \\
\hline \multicolumn{6}{|l|}{ Amount of anticoagulant use } \\
\hline$\leq 200 \mathrm{DDD}$ & 1.348 & $2.28(2.14-2.43)$ & $1.94(1.82-2.08)$ & $1.52(1.42-1.64)$ & $1.54(1.43-1.66)$ \\
\hline 200-1,100 DDD & 1.046 & $2.74(2.54-2.96)$ & $2.41(2.23-2.61)$ & $1.90(1.74-2.06)$ & $1.77(1.62-1.93)$ \\
\hline$>1,100 \mathrm{DDD}$ & 873 & $2.84(2.62-3.09)$ & $2.64(2.42-2.87)$ & $2.06(1.88-2.26)$ & $1.87(1.69-2.07)$ \\
\hline \multicolumn{6}{|l|}{ Duration of anticoagulant use } \\
\hline$\leq 2$ year & 1.867 & $2.47(2.33-2.62)$ & $2.12(1.99-2.25)$ & $1.61(1.51-1.72)$ & $1.57(1.47-1.68)$ \\
\hline $3-5$ years & 663 & $2.64(2.42-2.89)$ & $2.38(2.17-2.61)$ & $1.91(1.73-2.11)$ & $1.78(1.60-1.98)$ \\
\hline 6 or more years & 737 & $2.50(2.29-2.74)$ & $2.34(2.13-2.56)$ & $1.99(1.80-2.19)$ & $1.91(1.72-2.11)$ \\
\hline \multicolumn{6}{|l|}{ Intensity of anticoagulant use } \\
\hline$\leq 100 \mathrm{DDD} /$ year & 1.234 & $2.23(2.09-2.38)$ & $1.91(1.78-2.05)$ & $2.53(1.42-1.65)$ & $1.52(1.40-1.64)$ \\
\hline 97-207 DDD/year & 929 & $2.50(2.31-2.70)$ & $2.16(1.99-2.35)$ & $1.78(1.63-1.94)$ & $1.78(1.63-1.95)$ \\
\hline > 207 DDD/year & 1.104 & $3.09(2.87-3.34)$ & $2.84(2.62-3.06)$ & $2.07(1.90-2.25)$ & $1.83(1.67-2.01)$ \\
\hline \multicolumn{6}{|c|}{ Warfarin compared to anticoagulant non-users } \\
\hline None & 4.766 & Ref. & Ref. & Ref. & Ref. \\
\hline Ever-users & 1.549 & $2.34(2.20-2.48)$ & $2.06(1.94-2.19)$ & $1.73(1.63-1.85)$ & $1.66(1.55-1.77)$ \\
\hline \multicolumn{6}{|l|}{ Amount of warfarin use } \\
\hline$\leq 307 \mathrm{DDD}$ & 713 & $2.61(2.39-2.84)$ & $2.25(2.06-2.46)$ & $1.74(1.58-1.91)$ & $1.71(1.55-1.89)$ \\
\hline 307-1200 DDD & 482 & $2.29(2.07-2.55)$ & $2.19(1.97-2.44)$ & $1.85(1.66-2.07)$ & $1.76(1.57-1.98)$ \\
\hline$>1200$ DDD & 354 & $2.04(1.81-2.30)$ & $2.07(1.84-2.33)$ & $1.81(1.60-2.05)$ & $1.70(1.49-1.95)$ \\
\hline \multicolumn{6}{|l|}{ Duration of warfarin use } \\
\hline$\leq 2$ year & 720 & $2.45(2.25-2.67)$ & $2.18(1.99-2.38)$ & $1.70(1.55-1.87)$ & $1.67(1.51-1.84)$ \\
\hline $3-7$ years & 504 & $2.37(2.14-2.63)$ & $2.23(2.00-2.47)$ & $1.83(1.63-2.04)$ & $1.67(1.48-1.87)$ \\
\hline 8 or more years & 325 & $2.21(1.95-2.50)$ & $2.17(1.91-2.46)$ & $1.96(1.72-2.24)$ & $2.00(1.74-2.30)$ \\
\hline \multicolumn{6}{|l|}{ Intensity of warfarin use } \\
\hline$\leq 120$ DDD/year & 701 & $2.34(2.14-2.55)$ & $2.09(1.90-2.29)$ & $1.68(1.52-1.87)$ & $1.65(1.49-1.84)$ \\
\hline 120-193 DDD/year & 446 & $1.75(1.58-1.95)$ & $1.77(1.59-1.98)$ & $1.56(1.39-1.75)$ & $1.58(1.40-1.78)$ \\
\hline > 193 DDD/year & 402 & $1.53(1.37-1.70)$ & 1.75 (1.58-1.95) & $1.71(1.54-1.91)$ & $1.66(1.48-1.86)$ \\
\hline \multicolumn{6}{|c|}{ Warfarin compared to other anticoagulant drugs } \\
\hline Non-warfarin anticoagulant users & 1.718 & Ref. & Ref. & Ref. & Ref. \\
\hline Warfarin users & 1.549 & $0.45(0.41-0.50)$ & $1.00(0.91-1.09)$ & $1.09(0.99-1.21)$ & $1.03(0.92-1.14)$ \\
\hline \multicolumn{6}{|l|}{ Amount of warfarin use } \\
\hline$\leq 307 \mathrm{DDD}$ & 713 & $0.68(0.59-0.78)$ & $1.23(1.07-1.40)$ & $1.12(0.96-1.31)$ & $1.13(0.96-1.33)$ \\
\hline 307-1200 DDD & 482 & $0.39(0.33-0.45)$ & $0.94(0.83-1.07)$ & $1.10(0.96-1.26)$ & $0.99(0.86-1.15)$ \\
\hline$>1200$ DDD & 354 & $0.36(0.31-0.43)$ & $0.88(0.77-1.01)$ & $1.07(0.92-1.24)$ & $0.98(0.83-1.15)$ \\
\hline \multicolumn{6}{|l|}{ Duration of warfarin use } \\
\hline$\leq 2$ year & 720 & $0.58(0.50-0.66)$ & $1.17(1.03-1.33)$ & $1.11(0.96-1.28)$ & $1.09(0.93-1.27)$ \\
\hline $3-7$ years & 504 & $0.40(0.34-0.46)$ & $0.92(0.81-1.04)$ & $1.05(0.92-1.20)$ & $0.90(0.78-1.04)$ \\
\hline 8 or more years & 325 & $0.40(0.34-0.47)$ & $0.92(0.79-1.06)$ & $1.14(0.98-1.33)$ & $1.17(0.99-1.39)$ \\
\hline \multicolumn{6}{|l|}{ Intensity of warfarin use } \\
\hline$\leq 120 \mathrm{DDD} /$ year & 701 & $0.71(0.62-0.81)$ & $1.23(1.07-1.40)$ & $1.14(0.98-1.33)$ & $1.04(0.88-1.24)$ \\
\hline 120-193 DDD/year & 446 & $0.42(0.36-0.49)$ & $0.99(0.86-1.13)$ & $1.05(0.91-1.22)$ & $0.99(0.84-1.17)$ \\
\hline > 193 DDD/year & 402 & $0.31(0.27-0.37)$ & $0.86(0.76-0.98)$ & $1.09(0.96-1.25)$ & $1.04(0.91-1.20)$ \\
\hline \multicolumn{6}{|c|}{ LMWH compared to other anticoagulant drugs } \\
\hline Non-LMWH anticoagulant users & 1.534 & Ref. & Ref. & Ref. & Ref. \\
\hline LMWH users & 1.733 & $2.04(1.90-2.20)$ & $1.57(1.45-1.70)$ & $1.02(0.93-1.12)$ & $0.86(0.77-0.95)$ \\
\hline \multicolumn{6}{|l|}{ Amount of warfarin use } \\
\hline$\leq 40 \mathrm{DDD}$ & 497 & $1.09(0.98-1.20)$ & $0.99(0.89-1.11)$ & $0.81(0.72-0.91)$ & $0.73(0.63-0.83)$ \\
\hline
\end{tabular}


Table 2 (continued)

\begin{tabular}{|c|c|c|c|c|c|}
\hline & $n$ of deaths & Multivariable-adjusted & 1-year lag-time & 2-year lag-time & 3-year lag-time \\
\hline 40-80 DDD & 276 & $1.59(1.40-1.82)$ & $1.28(1.10-1.48)$ & $0.95(0.79-1.13)$ & $0.90(0.74-1.10)$ \\
\hline$>80 \mathrm{DDD}$ & 960 & $4.73(4.34-5.15)$ & $3.38(3.06-3.74)$ & $1.70(1.48-1.96)$ & $1.23(1.02-1.47)$ \\
\hline \multicolumn{6}{|c|}{ Duration of warfarin use } \\
\hline$\leq 1$ year & 1.173 & $1.80(1.66-1.94)$ & $1.46(1.34-1.59)$ & $0.96(0.87-1.06)$ & $0.83(0.74-0.93)$ \\
\hline 2 or more years & 560 & $3.00(2.71-3.32)$ & $2.00(1.77-2.27)$ & $1.29(1.09-1.52)$ & $0.98(0.79-1.21)$ \\
\hline \multicolumn{6}{|c|}{ Intensity of warfarin use } \\
\hline$\leq 40 \mathrm{DDD} /$ year & 580 & $1.12(1.01-1.23)$ & $1.01(0.91-1.11)$ & $0.82(0.73-0.92)$ & $0.75(0.65-0.85)$ \\
\hline 40-60 DDD/year & 198 & $1.66(1.43-1.93)$ & $1.35(1.14-1.61)$ & $1.07(0.87-1.32)$ & $0.97(0.77-1.23)$ \\
\hline > $60 \mathrm{DDD} /$ year & 955 & $4.83(4.43-5.26)$ & $3.40(3.07-3.75)$ & $1.62(1.41-1.87)$ & $1.15(0.96-1.38)$ \\
\hline
\end{tabular}

increase being strongest among those who had recorded diagnosis of VTE (Fig. 2).

When comparing warfarin to other types of anticoagulants, effect modification was observed for indication of anticoagulant use, Charlson Comorbidity Score and for NSAID, antihypertensive and antidiabetic drug use. Otherwise, the risk of overall death was similar independent of background variables (Fig. 3).

Among LMWH users compared to use of other anticoagulant drugs, effect modification was observed for VTE, atrial fibrillation, Charlson Comorbidity Score, and use of antihypertensive drugs (Supplementary Fig. 2)

\section{Sensitivity analysis}

Since the data included cancer deaths but not cancer diagnoses, we performed a sensitivity analysis comparing crude median survival times to end of year 2014 for the studied cancer types for anticoagulant non-user, anticoagulant users, and users of warfarin only and LMWH only. Median followup ranged from 0 to 4.5 years, being lowest for lung and hepatic cancer and highest for renal and bladder cancer and non-Hodgkin lymphomas. These data are provided in Supplementary Table.

\section{Discussion}

Anticoagulant use was associated with an increased risk of overall and cancer-specific cancer death independent of duration, amounts, or intensity of use. The risk increase prevailed although slightly attenuated in all lag-time analyses. The risk association was modified by indications of usage and by comorbidities; especially diagnosis of VTE greatly modified the risk association, although number of recorded VTE diagnoses was relatively low. For specific cancer types, the risk of cancer-specific death was increased for all cancer types, especially for bladder cancer.
The risk associations were similar when comparing users of warfarin to anticoagulant non-users for both overall and cancer-specific risk of death. When comparing warfarin users to users of other anticoagulant drugs, the risk was considerably lower in the non-lagged analysis but in the lag-time analyses the risk associations were similar. The same was observed for the risk of cancer-specific death. Thus, the timing of warfarin use modified the risk associations, especially when comparing to anticoagulant non-users. Strongest effect modification was observed by diagnosis of atrial fibrillation, Charlson Comorbidity Score, and use of antihypertensive drugs.

All anticoagulant sub-types were associated with an increased risk of cancer death. However, risk of cancer death among warfarin users compared to other anticoagulants was much lower but still elevated compared to nonusers although warfarin is not recommended for treatment of cancer-induced thrombi. A possible explanation for the lower risk among users of warfarin compared to other anticoagulants in the non-lagged analysis is most likely due to an increased risk associated with indication of anticoagulant use, especially LMWH use which was associated with significantly high risk of overall cancer death and cancer-specific death in the non-lagged analysis. LMWH is recommended for management of VTE in patients with cancer, as the likelihood of VTE recurrence may be lower when using this drug group in cancer patients [32]. Current Finnish guidelines for thromboprophylaxis in cancer patients recommend treatment with LMWH for 3-12 months or permanently. The vast majority (69\%) of the LMWH users in our study cohort used less than 80 DDD of LMWH, equaling a duration of use for less than 3 months.

Despite promising results in experimental animal studies [6-13], we found no decreased risk of cancer death associated with either warfarin or other anticoagulant drugs. On the contrary, the risk of cancer death is significantly increased. Out of previous epidemiological studies, our study is only comparable to O'Rorke et al. [20], as the other two studies [19,21] covered only prostate cancer 
Table 3 Multivariable and lag-time hazard ratios (95\% CI) related to cancer-specific deaths

\begin{tabular}{|c|c|c|c|c|c|}
\hline & $n$ of deaths & Multivariable-adjusted & 1-year lag-time & 2-year lag-time & 3-year lag-time \\
\hline \multicolumn{6}{|c|}{ Any anticoagulant compared to anticoagulant non-users } \\
\hline \multicolumn{6}{|l|}{ All cancers } \\
\hline Non-user & 4.766 & Ref. & Ref. & Ref. & Ref. \\
\hline Ever-user & 3.267 & $2.50(2.37-2.64)$ & $2.20(2.08-2.32)$ & $1.73(1.64-1.83)$ & $1.67(1-57-1.77)$ \\
\hline \multicolumn{6}{|l|}{ Cancer type } \\
\hline Lung cancer & $799 / 1,404^{\mathrm{a}}$ & $2.19(1.97-2.42)$ & $1.89(1.70-2.10)$ & $1.55(1.39-1.74)$ & $1.53(1.36-1.71)$ \\
\hline Gastric cancer & $124 / 203^{\mathrm{a}}$ & $3.03(2.33-3.96)$ & $2.93(2.24-3.85)$ & $2.13(2.60-2.84)$ & $2.00(1.48-2.70)$ \\
\hline Colorectal cancer & $350 / 414^{\mathrm{a}}$ & $3.51(2.96-4.16)$ & $3.01(2.53-3.58)$ & $2.15(1.80-2.58)$ & $1.80(1.49-2.18)$ \\
\hline Central nervous system cancers & $70 / 112^{\mathrm{a}}$ & $3.23(2.25-4.64)$ & $3.02(2.09-4.36)$ & $1.59(1.06-2.39)$ & $1.28(0.83-1.99)$ \\
\hline Non-Hodgkin lymphomas & $119 / 125^{\mathrm{a}}$ & $2.91(2.16-3.93)$ & $2.53(1.87-3.42)$ & $1.97(1.44-2.68)$ & $1.69(1.23-2.33)$ \\
\hline Hepatic cancer & $149 / 292^{\mathrm{a}}$ & $1.64(1.30-2.06)$ & $1.43(1.13-1.82)$ & $1.32(1.03-1.69)$ & $1.37(1.06-1.78)$ \\
\hline Pancreatic cancer & $301 / 431^{\mathrm{a}}$ & $2.90(2.44-3.46)$ & $2.10(1.75-2.52)$ & $1.48(1.22-1.80)$ & $1.51(1.23-1.84)$ \\
\hline Kidney cancer & $115 / 124^{\mathrm{a}}$ & $3.08(2.28-4.17)$ & $2.35(1.73-3.19)$ & $1.75(1.27-2.41)$ & $1.75(1.26-2.43)$ \\
\hline Bladder cancer & $108 / 87^{\mathrm{a}}$ & $4.99(3.58-6.96)$ & $4.44(3.19-6.20)$ & $2.87(2.04-4.04)$ & $2.67(1.89-3.77)$ \\
\hline \multicolumn{6}{|c|}{ Warfarin compared to anticoagulant non-users } \\
\hline \multicolumn{6}{|l|}{ All cancers } \\
\hline No anticoagulant use & 4.766 & Ref. & Ref. & Ref. & Ref. \\
\hline Warfarin ever-use & 1.549 & $2.34(2.20-2.48)$ & $2.06(1.94-2.19)$ & $1.73(1.63-1.85)$ & $1.66(1.55-1.77)$ \\
\hline \multicolumn{6}{|l|}{ Cancer type } \\
\hline Lung cancer & $380 / 1,404^{b}$ & $2.01(1.79-2.26)$ & $1.74(1.54-1.97)$ & $1.51(1.33-1.72)$ & $1.40(1.22-1.60)$ \\
\hline Gastric cancer & $52 / 203^{\mathrm{b}}$ & $2.89(2.14-3.90)$ & $2.56(1.87-3.51)$ & $2.02(1.45-2.82)$ & $1.85(1.30-2.63)$ \\
\hline Colorectal cancer & $144 / 414^{\mathrm{b}}$ & $3.10(2.56-3.76)$ & $2.68(2.20-3.26)$ & $2.03(1.65-2.49)$ & $1.82(1.47-2.25)$ \\
\hline Central nervous system cancers & $32 / 112^{\mathrm{b}}$ & $2.68(1.77-4.05)$ & $2.43(1.58-3.74)$ & $1.39(0.87-2.23)$ & $1.02(0.61-1.73)$ \\
\hline Non-Hodgkin lymphomas & $64 / 125^{\mathrm{b}}$ & $2.73(1.96-3.81)$ & $2.48(1.78-3.46)$ & $2.04(1.45-2.86)$ & $1.88(1.32-2.67)$ \\
\hline Hepatic cancer & $79 / 292^{b}$ & $1.47(1.13-1.91)$ & $1.51(1.16-1.96)$ & $1.42(1.08-1.87)$ & $1.53(1.15-2.02)$ \\
\hline Pancreatic cancer & $118 / 431^{\mathrm{b}}$ & $2.47(2.02-3.01)$ & $1.80(1.46-2.22)$ & $1.55(1.25-1.93)$ & $1.54(1.22-1.93)$ \\
\hline Renal cancer & $51 / 124^{\mathrm{b}}$ & $2.73(1.95-3.83)$ & $2.12(1.50-2.99)$ & $1.57(1.09-2.25)$ & $1.40(0.95-2.06)$ \\
\hline Bladder cancer & $52 / 87^{\mathrm{b}}$ & $5.29(3.69-7.60)$ & $4.45(3.09-6.40)$ & $2.91(2.00-4.24)$ & $2.62(1.78-3.88)$ \\
\hline \multicolumn{6}{|c|}{ Warfarin compared to other anticoagulant drugs } \\
\hline \multicolumn{6}{|c|}{ All cancers } \\
\hline Non-warfarin anticoagulant users & 1.718 & Ref. & Ref. & Ref. & Ref. \\
\hline Warfarin users & 1.549 & $0.45(0.41-0.50)$ & $1.00(0.91-1.09)$ & $1.09(0.99-1.21)$ & $1.03(0.92-1.14)$ \\
\hline \multicolumn{6}{|l|}{ Cancer type } \\
\hline Lung cancer & $380 / 419^{c}$ & $0.63(0.52-0.75)$ & $1.09(0.91-1.30)$ & $1.13(0.93-1.38)$ & $1.00(0.81-1.24)$ \\
\hline Gastric cancer & $52 / 72^{c}$ & $0.35(0.20-0.61)$ & $1.11(0.70-1.77)$ & $1.29(0.78-2.12)$ & $0.86(0.49-1.51)$ \\
\hline Colorectal cancer & $144 / 206^{\mathrm{c}}$ & $0.37(0.27-0.51)$ & $0.86(0.65-1.15)$ & $1.02(0.74-1.40)$ & $1.10(0.77-1.56)$ \\
\hline Central nervous system cancers & $32 / 38^{\mathrm{c}}$ & $0.43(0.23-0.83)$ & $1.40(0.77-2.55)$ & $1.08(0.52-2.26)$ & $1.00(0.44-2.27)$ \\
\hline Non-Hodgkin lymphomas & $64 / 55^{\mathrm{c}}$ & $0.48(0.29-0.78)$ & $1.79(1.14-2.82)$ & $1.75(1.07-2.87)$ & $1.64(0.96-2.81)$ \\
\hline Hepatic cancer & $70 / 79^{c}$ & $0.45(0.29-0.69)$ & $0.82(0.53-1.25)$ & $0.97(0.62-1.53)$ & $1.06(0.65-1.71)$ \\
\hline Pancreatic cancer & $118 / 183^{c}$ & $0.34(0.23-0.48)$ & $0.79(0.57-1.10)$ & $1.26(0.88-1.80)$ & $1.16(0.79-1.69)$ \\
\hline Renal cancer & $51 / 64^{\mathrm{c}}$ & $0.31(0.18-0.56)$ & $0.71(0.43-1.18)$ & $0.77(0.43-1.36)$ & $0.60(0.32-1.14)$ \\
\hline Bladder cancer & $52 / 56^{\mathrm{c}}$ & $0.41(0.23-0.72)$ & $0.98(0.59-1.63)$ & $1.16(0.66-2.03)$ & $1.40(0.79-2.51)$ \\
\hline \multicolumn{6}{|c|}{ LMWH compared to other anticoagulants } \\
\hline \multicolumn{6}{|l|}{ All cancers } \\
\hline Non-LMWH anticoagulants & 1.534 & Ref. & Ref. & Ref. & Ref. \\
\hline LMWH & 1.733 & $2.04(1.90-2.20)$ & $1.57(1.45-1.70)$ & $1.02(0.93-1.12)$ & $0.86(0.77-0.95)$ \\
\hline \multicolumn{6}{|l|}{ Cancer type } \\
\hline Lung cancer & $351 / 448^{\mathrm{d}}$ & $1.46(1.26-1.69)$ & $1.10(0.93-1.30)$ & $0.67(0.54-0.82)$ & $0.61(0.48-0.77)$ \\
\hline Gastric cancer & $48 / 76^{\mathrm{d}}$ & $3.39(2.30-4.98)$ & $3.19(2.14-4.75)$ & $1.31(0.82-2.09)$ & $1.16(0.69-1.96)$ \\
\hline Colorectal cancer & $233 / 117^{\mathrm{d}}$ & $3.60(2.84-4.56)$ & $2.75(2.15-3.50)$ & $1.70(1.29-2.23)$ & $1.06(0.77-1.45)$ \\
\hline
\end{tabular}


Table 3 (continued)

\begin{tabular}{|c|c|c|c|c|c|}
\hline & $n$ of deaths & Multivariable-adjusted & 1-year lag-time & 2-year lag-time & 3-year lag-time \\
\hline Central nervous system cancers & $27 / 43^{\mathrm{d}}$ & $1.10(0.66-1.84)$ & $0.73(0.41-1.31)$ & $0.65(0.30-1.40)$ & $0.76(0.32-1.79)$ \\
\hline Non-Hodgkin lymphomas & $65 / 54^{\mathrm{d}}$ & $2.38(1.62-3.50)$ & $2.17(1.44-3.26)$ & $1.87(1.20-2.94)$ & $1.79(1.09-2.93)$ \\
\hline Hepatic cancer & $67 / 82^{\mathrm{d}}$ & $1.46(1.04-2.05)$ & $0.84(0.56-1.25)$ & $0.43(0.25-0.73)$ & $0.45(0.25-0.80)$ \\
\hline Pancreatic cancer & $195 / 106^{\mathrm{d}}$ & $3.57(2.78-4.58)$ & $1.91(1.46-2.50)$ & $1.05(0.76-1.46)$ & $0.81(0.56-1.18)$ \\
\hline Renal cancer & $72 / 43^{\mathrm{d}}$ & $3.21(2.14-4.81)$ & $2.81(1.83-4.32)$ & $1.72(1.07-2.77)$ & $1.46(0.87-2.44)$ \\
\hline Bladder cancer & $66 / 42^{\mathrm{d}}$ & $2.80(1.85-4.25)$ & $1.78(1.16-2.73)$ & $0.95(0.57-1.59)$ & $0.67(0.37-1.21)$ \\
\hline
\end{tabular}

Comparison between (1) anticoagulant users and non-users, (2) warfarin users and anticoagulant non-users, (3) warfarin users and users of other anticoagulants, and (4) LMWH users and user of other anticoagulants

Statistically significant results are bolded

${ }^{a}$ Deaths among anticoagulant users/deaths among non-users

${ }^{b}$ Deaths among warfarin users/deaths among anticoagulant non-users

${ }^{c}$ Deaths among warfarin users/deaths among users of other anticoagulants

${ }^{\mathrm{d}}$ Deaths among LMWH users/death among users of other anticoagulants

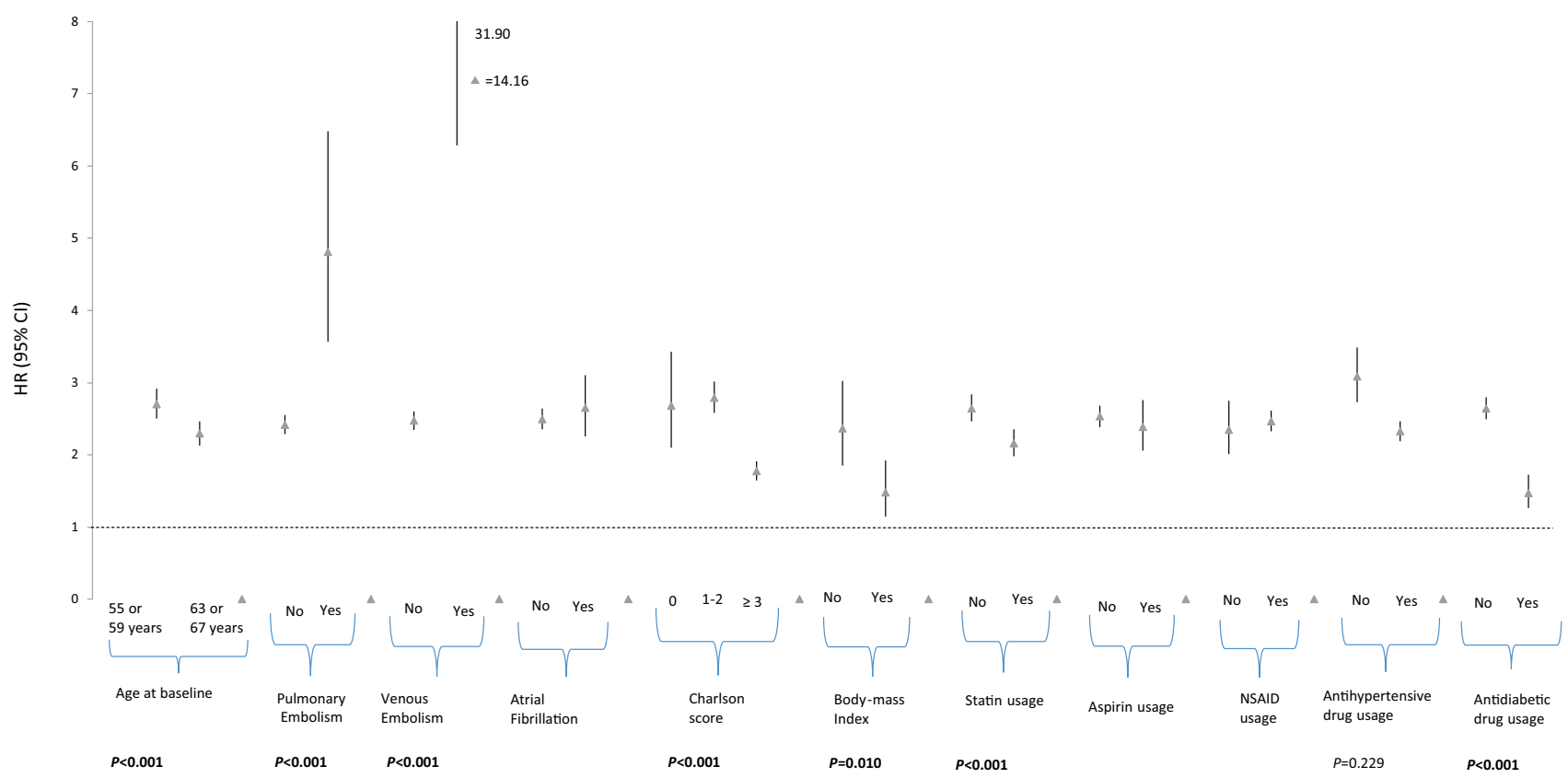

Fig. 2 Subgroup analysis between anticoagulant users and non-users for non-specific cancer death. $p$ for interaction is given under the variable if effect modification was considered possible. Statistically significant $p$ values are bolded. NSAID non-steroidal anti-inflammatory drugs

deaths. O'Rorke et al. additionally included lung, colorectal, and breast cancer-specific deaths. They reported increased risk of lung cancer death for pre-diagnostic but no risk increase for post-diagnostic warfarin use. For colorectal cancer, an increased risk of death was associated with post-diagnostic, but not with pre-diagnostic use. Our results for lung and colorectal cancer death and warfarin use are similar. Since we did not have sufficient data to divide anticoagulant use into pre- and post-diagnostic use, our results are not completely comparable, but both studies suggest that among lung and colorectal cancer patients, warfarin use is not associated with decreased mortality.

This study has several strengths: a large population-based cohort with a median follow-up of 17.2 years and detailed register-based information on anticoagulant use preventing recall bias. We were able to stratify use of anticoagulants by amounts (DDD), duration, and intensity of use, and to compare anticoagulant users with non-users, as well as warfarin users to users of other anticoagulants in addition to anticoagulant non-users. We also compared LMWH users to users 


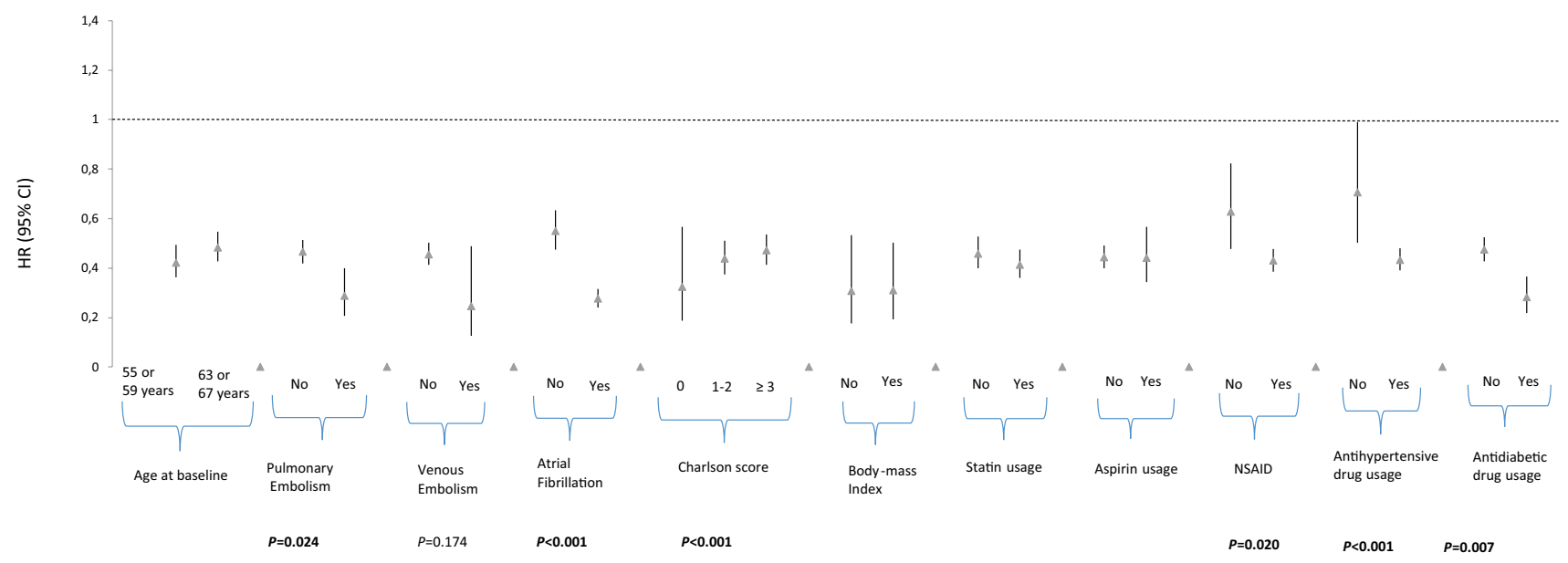

Fig. 3 Subgroup analysis between warfarin users and users of other types of anticoagulants for non-specific cancer death. $p$ for interaction is given under the variable if effect modification was considered pos-

of other anticoagulant drugs. Additionally, we were able to perform lag-time analyses to estimate the impact of timing of anticoagulant use. We were also able to study several cancer types to evaluate cancer-specific risk of death. For several cancer types, risk of cancer-specific death among anticoagulant users has not been reported earlier in epidemiological studies. Most published clinical trials on this topic have involved breast cancer and lung cancer patients. Prostate cancer was not separately analyzed in this study, as we have previously covered it in detail [21]. In that study, we reported that post-diagnostic use of anticoagulants was associated with 1.6-fold and use of warfarin 1.5-fold risk of prostate cancer death when compared to anticoagulant non-users.

This study also has certain limitations. For many cancer types, the number of deaths was relatively low. We had limited information on BMI, only for 11,345 men (15\%), which may influence cancer mortality and cause confounding [33]. BMI was assessed only in the subgroup analysis. Additionally, we did not have information on smoking, which has been associated with increased risk of death in many cancer types [34]. However, we were able to adjust for Charlson Comorbidity Score. We did not have information on dietary factors and physical activity which are linked to cancer prognosis [35]. Misclassification in cause of death on death certificates has been reported which could bias the association away from null [36]. Another limitation is that drug use during hospital inpatient periods is not covered. However, if a person has had a clear indication for anticoagulant usage, i.e., not only prophylaxis, the drug use is continued after hospitalization and is visible in the data used in this study. Furthermore, our study was not randomized and hence the comparability of the users and non-users was uncertain, with potential for confounding by indication. Additionally, sible. Statistically significant $p$ values are bolded. NSAID non-steroidal anti-inflammatory drugs

our results might not be applicable to younger population, women, or non-Caucasian ethnicities, as this cohort covered only Finnish men aged 55-67 at baseline.

In a population-based setting, use of anticoagulants is associated with an increased risk of cancer death. The risk increase is likely caused by increased likelihood of thrombosis in cancer patients and the resulting treatment. In warfarin users, the risk increase was smaller compared to users of LMWH, but the risk was nevertheless increased compared to non-users of anticoagulants.

\section{Conclusion}

Anticoagulants as a group are associated with an increased risk of cancer death. Our study does not support reduced cancer mortality among anticoagulant drug users. For future studies exploring use of any drug group and cancer mortality, we recommend adjusting for use of anticoagulant drugs as this drug group is rarely adjusted for and is associated with significantly higher risk of cancer death. Additionally, we recommend exploring new oral anticoagulants and cancer mortality as they have now been in use for several years, but epidemiological studies on the topic are virtually non-existing.

Acknowledgments Supported by non-restricted competitive research funding from the Pirkanmaa Hospital District 150640 to TJ Murtola and non-restricted research grant from Pirkanmaa Cancer Society to PTT Kinnunen. Academy of Finland (grant 132385 and 260 931) and Cancer Organisations of Finland to A Auvinen, Competitive Research funding of Pirkanmaa Hospital District to TLJ Tammela. No funder organization participated in any part of the study design or collection, analysis or interpretation of data, or writing the manuscript. 
Data availability Permission to use the entire data has been granted personally for this specific study, and thus we are not allowed to make the entire data publicly available without permission. Limited data (i.e., variables used for the analyses) can be obtained upon reasonable request. Each Administrator and Information Commissioner of the used registries processed our request to use the data and granted a personal permission. If desired, similar permissions can be applied from the Finnish Social Insurance Institution (available at http://www. kela.fi/web/en/research) and the National Institute for Health and Welfare (available at https://www.thl.fi/en/web/thlfi-en/statistics/informatio n-for-researchers).

\section{Compliance with ethical standards}

Competing interests We declare the following competing interestsPTT Kinnunen and K Talala: no competing interests. TJ Murtola: paid consultant for Astellas and Janssen-Cilag, and lecture fees from Astellas, Janssen-Cilag, Abbvie, and MSD. K Taari: research funding from Medivation, Astellas, Pfizer, and Orion, and travel support from Astellas. TLJ Tammela: paid consultant for Astellas, Orion Pharma, and Janssen-Cilag. A Auvinen: lecture fee from MSD, and paid consultant for Epid Research Inc.

Ethics approval The study has been approved by the Ethical Committee of Pirkanmaa Hospital District (Committee's reference number $\mathrm{R} 10167)$. This study is based on register data collected routinely for other purposes. Thus, no informed consent is needed based on international practices.

Open Access This article is distributed under the terms of the Creative Commons Attribution 4.0 International License (http://creativeco mmons.org/licenses/by/4.0/), which permits unrestricted use, distribution, and reproduction in any medium, provided you give appropriate credit to the original author(s) and the source, provide a link to the Creative Commons license, and indicate if changes were made.

\section{References}

1. Blom JW, Doggen CJM, Osanto S, Rosendaal FR (2005) Malignancies, prothrombotic mutations, and the risk of venous thrombosis. JAMA 293:715-722

2. Walker AJ, Card TR, West J, Crooks C, Grainge MJ (2013) Incidence of venous thromboembolism in patients with cancer-a cohort study using linked United Kingdom databases. Eur J Cancer 49:1404-1413

3. Cronin-Fenton DP, Sondergaard F, Pedersen LA et al (2010) Hospitalisation for venous thromboembolism in cancer patients and the general population: a population-based cohort study in Denmark, 1997-2006. Br J Cancer 103:947-953

4. Prandoni P, Lensing AW, Prins MR (1997) The natural history of deep-vein thrombosis. Semin Thromb Hemost 23:185-188

5. Sørensen HT, Mellemkjaer L, Olsen JH, Baron JA (2000) Prognosis of cancers associated with venous thromboembolism. N Engl J Med 343:1846-1850

6. Nierodzik ML, Plotkin A, Kajumo F, Karpatkin S (1991) Thrombin stimulates tumor-platelet adhesion in vitro and metastasis in vivo. J Clin Invest 87:229-236

7. Kondraganti S, Gondi CS, Gujrati M et al (2006) Restoration of tissue factor pathway inhibitor inhibits invasion and tumor growth in vitro and in vivo in a malignant meningioma cell line. Int $\mathbf{J}$ Oncol 29:25-32
8. Ichikawa J, Cole HA, Magnussen RA et al (2012) Thrombin induces osteosarcoma growth, a function inhibited by low molecular weight heparin in vitro and in vivo: procoagulant nature of osteosarcoma. Cancer 118:2494-2506

9. Bruggemann LW, Versteeg HH, Niers TM, Reitsma PH, Spek CA (2008) Experimental melanoma metastasis in lungs of mice with congenital coagulation disorders. J Cell Mol Med 12:2622-2627

10. Degen JL, Palumbo JS (2012) Hemostatic factors, innate immunity and malignancy. Thromb Res 129:S1-S5

11. Horowitz NA, Blevins EA, Miller WM et al (2011) Thrombomodulin is a determinant of metastasis through a mechanism linked to the thrombin binding domain but not the lectin-like domain. Blood 118:2889-2895

12. Amirkhosravi A, Mousa SA, Amaya M, Francis JL (2003) Antimetastatic effect of tinzaparin, a low-molecular-weight heparin. J Thromb Haemost 1:1972-1976

13. Hejna M, Raderer M, Zielinski CC (1999) Inhibition of metastases by anticoagulants. J Natl Cancer Inst 91:22-36

14. Maat B (1980) Selective macrophage inhibition abolishes warfarin-induced reduction of metastasis. Br J Cancer 41:313-316

15. Brown JM (1973) A study of the mechanism by which anticoagulation with warfarin inhibits blood-borne metastases. Cancer Res 33:1217-1224

16. Smorenburg SM, Vink R, Otten HM, Swaneveld F, Buller HR (2001) The effects of vitamin K-antagonists on survival of patients with malignancy: a systematic analysis. Thromb Haemost $86: 1586-1587$

17. Gould MK, Dembitzer AD, Doyle RL, Hastie TJ, Garber AM (1999) Low-molecular-weight heparins compared with unfractionated heparin for treatment of acute deep venous thrombosis. A meta-analysis of randomized, controlled trials. Ann Intern Med 130:800-809

18. Sanford D, Naidu A, Alizadeh N, Lazo-Langner A (2014) The effect of low molecular weight heparin on survival in cancer patients: an updated systematic review and meta-analysis of randomized trials. J Thromb Haemost 12:1076-1085

19. Akl EA, Kahale LA, Ballout RA et al (2014) Parenteral anticoagulation in ambulatory patients with cancer. Cochrane Database Syst Rev 12:006652

20. Kuderer NM, Khorana AA, Lyman GH, Francis CW (2007) A meta-analysis and systematic review of the efficacy and safety of anticoagulants as cancer treatment: impact on survival and bleeding complications. Cancer 110:1149-1161

21. Tagalakis V, Tamim H, Blostein M, Hanley JA, Kahn SR (2013) Risk of prostate cancer death in long-term users of warfarin: a population-based case-control study. Cancer Causes Control 24:1079-1085

22. O'Rorke MA, Murray LJ, Hughes CM, Cantwell MM, Cardwell CR (2015) The effect of warfarin therapy on breast, colorectal, lung, and prostate cancer survival: a population-based cohort study using the Clinical Practice Research Datalink. Cancer Causes Control 26:355-366

23. Kinnunen PTT, Murtola TJ, Talala K, Taari K, Tammela TLJ, Auvinen A (2017) Prostate cancer-specific survival among warfarin users in the Finnish Randomized Study of Screening for Prostate Cancer. BMC Cancer 17:585

24. Kilpelainen TP, Tammela TL, Malila N et al (2013) Prostate cancer mortality in the Finnish randomized screening trial. J Natl Cancer Inst 105:719-725

25. Charlson ME, Pompei P, Ales KL, MacKenzie CR (1987) A new method of classifying prognostic comorbidity in longitudinal studies: development and validation. J Chronic Dis 40:373-383

26. Zhong S, Zhang X, Chen L, Ma T, Tang J, Zhao J (2015) Statin use and mortality in cancer patients: systematic review and meta-analysis of observational studies. Cancer Treat Rev 41:554-567 
27. Mills EJ, Wu P, Alberton M, Kanters S, Lanas A, Lester R (2012) Low-dose aspirin and cancer mortality: a meta-analysis of randomized trials. Am J Med 125:560-567

28. Liu Y, Chen J, Xie L et al (2014) Effect of aspirin and other nonsteroidal anti-inflammatory drugs on prostate cancer incidence and mortality: a systematic review and meta-analysis. BMC Med $12: 55$

29. Harding JL, Sooriyakumaran M, Anstey KJ et al (2016) Hypertension, antihypertensive treatment and cancer incidence and mortality: a pooled collaborative analysis of 12 Australian and New Zealand cohorts. J Hypertens 34:149-155

30. Yin M, Zhou J, Gorak EJ, Quddus F (2013) Metformin is associated with survival benefit in cancer patients with concurrent type 2 diabetes: a systematic review and meta-analysis. Oncologist $18: 1248-1255$

31. WHO ATC/DDD index 2016. http://www.whocc.no/atc_ddd_ index/. Accessed 20 Dec 2016

32. Venous thromboembolic diseases: the management of venous thromboembolic diseases and the role of thrombophilia testing. Clinical guideline. National Clinical Guideline Center (NCGC) 2012
33. Taghizadeh N, Boezen HM, Schouten JP, Schroder CP, Elisabeth de Vries EG, Vonk JM (2015) BMI and lifetime changes in BMI and cancer mortality risk. PLoS ONE 10:e0125261

34. Meyer J, Rohrmann S, Bopp M, Faeh D, Swiss National Cohort Study Group (2015) Impact of smoking and excess body weight on overall and site-specific cancer mortality risk. Cancer Epidemiol Biomark Prev 24:1516-1522

35. Whiteman DC, Wilson LF (2016) The fractions of cancer attributable to modifiable factors: a global review. Cancer Epidemiol 44:203-221

36. Hinchliffe SR, Abrams KR, Lambert PC (2013) The impact of under and over-recording of cancer on death certificates in a competing risks analysis: a simulation study. Cancer Epidemiol 37:11-19

Publisher's Note Springer Nature remains neutral with regard to jurisdictional claims in published maps and institutional affiliations. 